\title{
3D Surface Reconstruction of a Moving Object in the Presence of Specular Reflection
}

\author{
Atsuto Maki \\ Graduate School of Informatics, Kyoto University, \\ Kyoto, 606-8501, Japan
}

\begin{abstract}
We present a new scheme for $3 \mathrm{D}$ surface reconstruction of a moving object in the presence of specular reflection. We basically search for the depth at each point on the surface of the object while exploiting the recently proposed geotensity constraint 7 that accurately governs the relationship between four or more images of a moving object in spite of the illumination variance due to object motion. The thrust of this paper is then to extend the availability of the geotensity constraint to the case that specularities are also present. The key idea is to utilise the fact that highlights shift on the surface due to object motion. I.e., we employ five or more images as inputs, and interchangeably utilise a certain intensity subset consisting of four projected intensities which is the least influenced by the specular component. We illustrate the relevancy of our simple algorithm also through experiments.
\end{abstract}

\section{Introduction}

Given a set of images, in each of which an object is viewed from a different direction, the fundamental issue in extracting 3D information of the object out of $2 \mathrm{D}$ images is to match corresponding points in those images so that these points are the projections of an identical point on the surface of it. For the point correspondence, typically exploited is the constraint that the corresponding parts of the images have equivalent image intensities, regarding the variation in illumination as noise. It has been successfully applied to stereo (see for example 45]) where two images are taken simultaneously as the lighting of the object is identical in each image. However, when we consider replacing the stereo camera with a single camera observing an object in motion, unfortunately the constraint is nearly always invalid as non-uniform lighting causes the intensity at a specific location on the surface of an object to change as the object moves. Among the few efforts for this issue, whereas photometric motion 9] treated the illumination variance due to object motion in terms of optical flow, geotensity constraint 1 [7] has been recently derived to overcome the problem with respect to camera geometry, and to replace the constant intensity constraint. Based on the notion of linear intensity subspaces [10, the geotensity constraint governs the relationship between four (or more) images of a moving object, and it can be computed and applied automatically to the task of 3D surface reconstruction.

\footnotetext{
${ }^{1}$ Geotensity stands for "geometrically corresponding pixel intensity."
} 
The algorithm for surface reconstruction using geotensity constraint proceeds basically in two stages. The first stage is to derive the parameters of the geotensity constraint by analysing coordinates and image intensities of some sample points on the object in motion. That is, computing structure from motion obtains the geometric parameters of the situation, whereas computing the linear image subspace obtains the lighting parameters of the situation. By combining both sets of parameters we arrive at the geotensity constraint. Using the same set of images, the second stage is to take each pixel in an arbitrary reference image in turn and search for the depth along the ray from the optical centre of the camera passing through the pixel. The depth is evaluated by measuring the agreement of the entire set of projected intensities of a point on the object surface with the geotensity constraint.

Although the availability of the constraint was limited in principle to Lambertian surface as is the case with the other sophisticated approaches [11 13, the thrust of this paper is to extend it to the situation that the object surface partly takes on specular reflection. In the case of stereo, in the presence of specular reflection, it has been shown possible to determine trinocular configurations such that at least one stereo pair can provide correct depth estimate at each scene point visible to all cameras [3. In contrast, given a single static camera, we propose to employ five or more images as inputs, and interchangeably utilise a certain intensity subset consisting of four projected image intensities that is the least influenced by the specular component of surface reflection. The strategy is motivated by the fact that the specularities shift on the surface thanks to the object motion. Through experiments we show that the proposed scheme indeed improves the depth estimate compared to the results by other standard algorithms in terms of dealing with specular reflection.

\section{Preliminaries}

As the background we first consider some issues respecting geometry and image intensity that form the basis of the geotensity constraint. What we initially need is to find some number of corresponding sample points by an independent mechanism as seen for example in 114]. Given point correspondence for some sample points, we can derive a constraint on geometry by the coordinates, and also a photometric constraint by observing the intensities on these points.

Solving for geometry. In this paper, for simplicity, we will concern ourselves with the affine and scaled-orthographic camera models for projection. Consider the $i^{\text {th }}$ world point $\mathbf{X}_{i}=\left(X_{i}, Y_{i}, Z_{i}\right)^{\top}$ on the surface of an object projected to image point $\mathbf{x}_{i}(j)=\left(x_{i}(j), y_{i}(j)\right)^{\top}$ in the $j^{\text {th }}$ frame. The affine camera model defines this projection to be $\mathbf{x}_{i}(j)=\mathbf{M}(j) \mathbf{X}_{i}+\mathbf{t}(j)$, where $\mathbf{M}(j)$, an arbitrary $2 \times 3$ matrix, and $\mathbf{t}(j)$, an arbitrary 2 vector, encode the motion parameters of the object. The solution to the structure from motion problem using singular value decomposition is well known for this case; given at least four point trajectories $\mathbf{x}_{i}(j)$ observed through at least two frames the set $\mathbf{M}(j), \mathbf{X}_{i}$ and $\mathbf{t}(j)$ can be uniquely recovered up to an arbitrary affine ambiguity [12]. The result is affine structure. 
Given the solution to structure from motion using the affine camera model, the Euclidean structure and motion parameters fitting the weak perspective camera model can be recovered. A result of choosing the first frame to be canonical is that the structure vectors have the form, $\mathbf{X}_{i}=\left(\mathbf{x}_{i}^{\top}(1), Z\right)^{\top}$, and we can derive the relationship which effectively describes the epipolar constraint between two images:

$$
\mathbf{x}_{i}(j)=\mathbf{M}(j)\left(\begin{array}{c}
\mathbf{x}_{i}(1) \\
Z
\end{array}\right)+\mathbf{t}(j) .
$$

Solving for image intensity. Assuming a static camera and a single point light source, we consider the intensity $I_{i}(j)$ of the $i^{\text {th }}$ point on the surface of a moving object projected into the $j^{\text {th }}$ image. For Lambertian surface, since the intensity can be equivalently represented as if it were generated on the surface of a static object under inversely moving point light source, we can then express $I_{i}(j)$ in terms of the image formation equation process so that

$$
I_{i}(j)=\max \left(\mathbf{b}_{i}^{\top} \mathbf{s}(j), 0\right) .
$$

The 3 -vector $\mathbf{b}_{i}$ is defined at the first frame to be the product of the albedo with the inward facing unit normal for the $i^{t h}$ point whereas the 3 -vector $\mathbf{s}(j)$ is the product of the strength of the light source with the unit vector for its direction. Note that $\mathbf{s}(j)=\mathbf{R}^{\top}(j) \mathbf{s}(1)$, where the $3 \times 3$ matrix, $\mathbf{R}(j)$, is the rotation of the object from the first frame, which is taken to be the reference, to the $j^{\text {th }}$ frame. Multiplication of $\mathbf{R}^{\top}(j)$ represents virtually inverse rotation of the light source. The rotation matrix is directly computed from the $2 \times 3$ matrix $\mathbf{M}(j)$ that is given above by solving for the structure from motion problem. Although the maximum operator zeroes negative components [6] which correspond to the shadowed surface points, we assume that there are no shadows in the subsequent formulations.

Given the correspondence for $n$ feature points through $m$ images, we record the corresponding pixel intensities, $I_{i}(j)$, in an $n \times m$ matrix as $\mathbf{I}=\left(I_{i}(j)\right)$, which we call the illumination matrix. Then, we can form the matrix equation, $\mathbf{I}=\mathbf{B S}$, where $\mathbf{B}$ is an $n \times 3$ matrix containing the rows $\mathbf{b}_{i}^{\top}$, and $\mathbf{S}$ is a $3 \times m$ matrix containing the columns $\mathbf{s}(j)$. Then, the familiar form for solution by singular value decomposition to obtain a rank 3 approximation to the matrix $\mathbf{I}$ is such that

$$
\mathbf{I}=\breve{\mathbf{B}} \breve{\mathbf{S}}=\left(\breve{\mathbf{B}} \mathbf{A}^{-1}\right)(\mathbf{A} \breve{\mathbf{S}})
$$

As is well known, the solution is unique up to an arbitrary invertible $3 \times 3$ transformation $\mathbf{A}$ which transforms $\breve{\mathbf{S}}$ into $\mathbf{S}$, or $\breve{\mathbf{s}}(j)$ into $\mathbf{s}(j)$, for each column by

$$
\mathbf{S}=\mathbf{A} \breve{\mathbf{S}}, \quad \mathbf{s}(j)=\mathbf{A} \breve{\mathbf{s}}(j),
$$

where $\breve{\mathbf{s}}(j)$ denotes each column of $\breve{\mathbf{S}}$.

\section{The Geotensity Constraint for Correspondence Search}

Although a thorough description of the geotensity constraint can be found in [7, in this section we briefly review the constraint while reformulating it so as to 


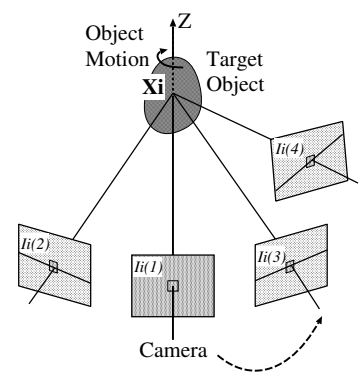

Fig. 1. Geotensity constraint. The intensity of world point $\mathbf{X}_{i}$ projected into the first image, $I_{i}(1)$, is represented by a unique linear combination of the intensities of the same point projected in the other three images, $I_{i}(2) \cdots I_{i}(4)$ for all points $i$.

prepare for the extension for the case with specularities which we will introduce in Section 4. The term geotensity constraint accounts for a constraint between four or more images of an object from different views under static lighting conditions. This concept is schematically depicted in Figure 1 by replacing object motion with an imaginary, and coherent motion of the camera and the light source.

The conditions for applying the geotensity constraint to surface reconstruction are as follows: (i) The scene consists of a single moving object that is convex (therefore no self-shadowing). (ii) There is a single distant light source. (iii) The object has Lambertian surface properties while the surface may or may not be textured. However, the condition (iii) will be relaxed in Section 4 .

Evaluating the set of intensities for correspondence. At each pixel, $x$, in the first image, to search for the depth $Z$ we can recall equation 1 for the geometric constraint imposed on a sequence of images so that

$$
I(j ; \mathbf{x}, Z)=I[\mathbf{x}(j)]=I\left[\mathbf{M}(j)\left(\begin{array}{c}
\mathbf{x}(1) \\
Z
\end{array}\right)+\mathbf{t}(j)\right] .
$$

$I(j ; \mathbf{x}, Z)(j=1, \ldots, m ; m \geq 4)$ indicates the set of image intensities in the $j^{\text {th }}$ frame at the coordinates determined by $\mathbf{x}$ in the first image, guess of depth $Z$, and the motion parameters $\mathbf{M}(j)$ and $\mathbf{t}(j)$. The task is now to evaluate the set of intensities $I(j ; \mathbf{x}, Z)$. If full Euclidean lighting conditions have been recovered in advance so that $\mathbf{s}(j)$ is known without ambiguity, we define $\hat{\mathbf{b}}^{\top}$ as

$$
\hat{\mathbf{b}}^{\top}=[I(1), \ldots, I(m)] \mathbf{S}^{\top}\left(\mathbf{S S}^{\top}\right)^{-1}
$$

where $\mathbf{S}$ is a $3 \times m$ matrix containing the columns $\mathbf{s}(j)(j=1, \ldots, m)$. For a single light source with all images taken with the light source in the so-called bright cell2 2 , the estimated values of the intensities would then be

$$
\hat{I}(j ; \mathbf{x}, Z)=\hat{\mathbf{b}}^{\top} \mathbf{s}(j) .
$$

$\overline{2}$ The cell of light source directions that illuminate all points on the object 2 . 
It should be noted that exactly the same estimation of $\hat{I}(j ; \mathbf{x}, Z)$ is available also in the case that the light source direction is determined only up to the ambiguity. This is easily confirmed by substituting equation 4 to equation 6, and then to equation [7, where matrix A turns out to be cancelled. Thus, the set of equations 6 and 7 can be represented equivalently as

$$
\hat{I}(j ; \mathbf{x}, Z)=\hat{\mathbf{b}}^{\top} \breve{\mathbf{s}}(j), \quad \hat{\mathbf{b}}^{\top}=[I(1), \ldots, I(m)] \breve{\mathbf{S}}^{\top}\left(\breve{\mathbf{S}}^{\top}\right)^{-1} .
$$

Estimating $\hat{I}(j ; \mathbf{x}, Z)$ by equation 8 , we can define the error function to evaluate the set of intensities $I(j ; \mathbf{x}, Z)$ as

$$
E(\mathbf{x}, Z)=\sum_{j=1}^{m}(I(j ; \mathbf{x}, Z)-\hat{I}(j ; \mathbf{x}, Z))^{2} .
$$

Computing the depth. At each pixel, $\mathbf{x}$, in the first reference image we measure the error, $E$, in the geotensity constraint at regular small intervals of depth, $Z$. When the depth is correct we expect the error to approach zero and when it is incorrect we expect the error to be large. The geotensity constraint can be therefore stated simply as $E(\mathbf{x}, Z)=0$. It is clear that as the depth parameter is varied the location of the corresponding points in each image will trace out the corresponding epipolar line in each image. We then choose such depth $Z$ that minimises the error $E(\mathbf{x}, Z)$ as the depth estimate.

\section{Dealing with Specular Reflection}

The technique of evaluating the set of projected intensities discussed in the previous section is deficient in dealing with specular reflection. This is because the notion of linear image basis which plays the essential role is only valid for Lambertian surface. In order to cope with specular reflection within the same framework, we propose to employ five or more images as inputs, and interchangeably utilise a certain subset consisting of four intensities, which are required at the minimum for the geotensity constraint to be applied.

Since we consider a moving object as the target, each surface point may or may not have specularity depending on the object's pose as a specular lobe in general has some certain limited directional range. We may thus expect that a certain subset of projected intensities exists that satisfies the geotensity constraint to a reasonable extent. At least it is very unlikely that some specular reflection is added to an identical surface point throughout all the input image frames (except the case the object consists of a particular kind of material such as metal). The problem to cope with the situation is then attributed to that of finding an intensity subset which is the least influenced by specular component of surface reflection.

Algorithm description. As the simplest case, let us consider that five images are given as input so that $m=5$, and assume that specular reflection occurs mostly in either of them due to object motion. Given an $n \times m$ illumination 
matrix as $\mathbf{I}=\left(I_{i}(j)\right)$, we define $m$ different $n \times(m-1)$ illumination submatrices, $\mathbf{I}_{\bar{k}}(k=1, \ldots, m)$, by skipping the $k^{t h}$ column, $\mathbf{I}(k)$, such as

$$
\mathbf{I}_{\bar{k}}=[\mathbf{I}(1) \ldots \mathbf{I}(k-1) \mathbf{I}(k+1) \ldots \mathbf{I}(m)] .
$$

We then recall equation 3 and compute a rank 3 approximation to each $\mathbf{I}_{\bar{k}}$,

$$
\mathbf{I}_{\bar{k}}=\breve{\mathbf{B}}_{\bar{k}} \breve{\mathbf{S}}_{\bar{k}}=\left(\breve{\mathbf{B}}_{\bar{k}} \mathbf{A}^{-1}\right)\left(\mathbf{A} \breve{\mathbf{S}}_{\bar{k}}\right)
$$

where $\breve{\mathbf{S}}_{\bar{k}}(k=1, \ldots, m)$ in this case is a $3 \times(m-1)$ matrix containing the columns $\breve{\mathbf{s}}(j)(j=1, \ldots, m-1)$. Importantly, for computing each $\breve{\mathbf{S}}_{\bar{k}}$ we employ RANSAC, a robust random sampling and consensus technique, to ensure that artifacts which are caused by specularities (or some intensities not fulfilling the assumed conditions, e.g. self-shadowing) do not distort the correct solution.

At the correct depth in correspondence search, an intensity subset, $I(j ; \mathbf{x}, Z)(j=$ $1, \ldots, m-1)$, excluding the $k^{t h}$ element should be properly validated with corresponding light source matrix $\breve{\mathbf{S}}_{\bar{k}}$ if specular reflection occurs in the $k^{\text {th }}$ frame. Hence, with each $\breve{\mathbf{S}}_{\bar{k}}$, we can exploit equation 8 to estimate $\hat{I}(j ; \mathbf{x}, Z)(j=$ $1, \ldots, m-1)$ and then evaluate the subset of intensities by

$$
E_{\bar{k}}(\mathbf{x}, Z)=\sum_{j=1, j \neq k}^{m}(I(j ; \mathbf{x}, Z)-\hat{I}(j ; \mathbf{x}, Z))^{2} .
$$

Namely, we obtain $m$ different $E_{\bar{k}}(\mathbf{x}, Z)(k=1, \ldots, m)$ due to $m$ different candidates of intensity subset (together with corresponding $\breve{\mathbf{S}}_{\bar{k}}$ ) and one of them must be appropriate according to the above assumption.

In order to search for the depth $Z$, we need to judge the correct error among those defined by equation 12 . Although it is not possible to know which subset of projected intensities is the least influenced by specularity in advance, since the error $E_{\bar{k}}(\mathbf{x}, Z)$ generally becomes larger with specularity, it is sensible to choose the smallest one so that

$$
E(\mathbf{x}, Z)=\min _{k} E_{\bar{k}}(\mathbf{x}, Z)
$$

Just as in the case without specular reflection, we expect the error to approach zero when the depth is correct, and choose such depth $Z$ that minimises the error $E(\mathbf{x}, Z)$ as the depth estimate. Our algorithm for estimating the depth in the presence of specularities can be summarised as following:

$1^{\circ}$ Decompose each illumination submatrix $\mathbf{I}_{\bar{k}}$ using SVD and yield $\breve{\mathbf{S}}_{\bar{k}}$. $2^{\circ}$ At point $\mathbf{x}$, measure $I(j ; \mathbf{x}, Z)$ by equation 5 for a guess of depth $Z$. $3^{\circ}$ Estimate $\hat{I}(j ; \mathbf{x}, Z)$ by equation 8 , for all $\breve{\mathbf{S}}_{\bar{k}}$ using $I(j ; \mathbf{x}, Z)(j \neq k)$.

$4^{\circ}$ Compute $E_{\bar{k}}(\mathbf{x}, Z)$ and then $E(\mathbf{x}, Z)$ by equations 12 and 13 .

$5^{\circ}$ Choose such depth $Z$ that minimises $E(\mathbf{x}, Z)$ as the depth estimate.

Alternatively, we may simplify step $3^{\circ}$ by estimating $\hat{I}(j ; \mathbf{x}, Z)$ just in one way, rather than examining it for all the possible candidates of $\breve{\mathbf{S}}_{\bar{k}}$. That is, we simply 

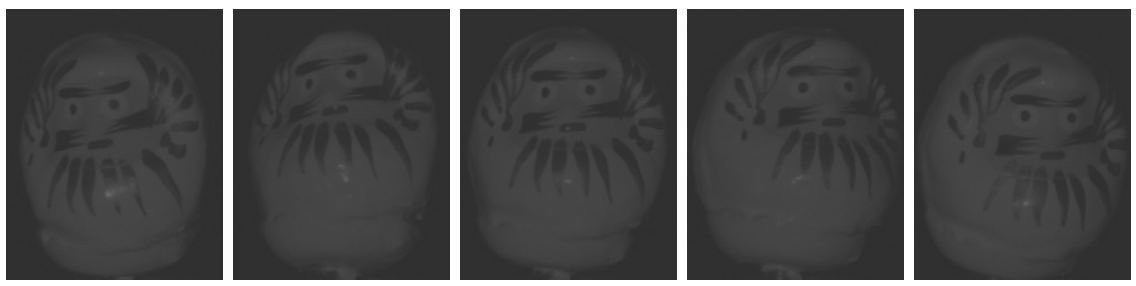

Fig. 2. Example input images of a Dharma doll. Captured in different poses under a point light source placed beside the camera (Nikon D70). Image size: $320 \times 400$ pixels. It is observed that the specularities shift on the surface thanks to the object motion.
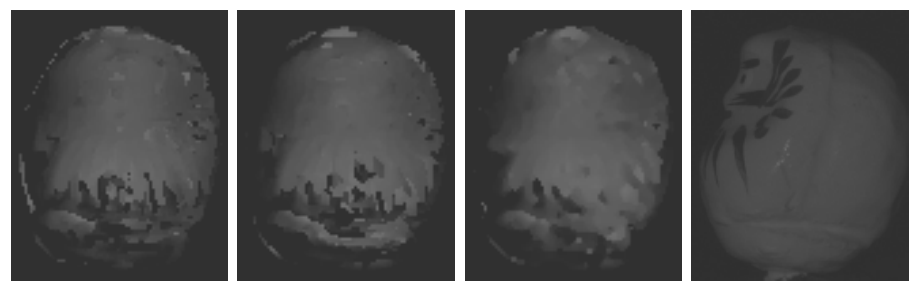

Fig. 3. Three depth maps estimated referring the midmost image in Figure 2 (the lighter, the closer). From the left: differencing, the geotensity, and the geotensity with intensity subset. The profile of the Dharma doll is also shown for reference.

determine $k$ such that the $k^{t h}$ element has the highest value among the set of projected intensities, and compute $E_{\bar{k}}(\mathbf{x}, Z)$ accordingly. It is based on the observation that setting the element of highest intensity aside must be the choice for the solution to be the least influenced by specular reflection at the correct depth. As we can then skip estimating different $E_{\bar{k}}(\mathbf{x}, Z)$ in step $4^{\circ}$, it is also less expensive in the computational viewpoint.

Finally, the basic principle in the proposition applies to a larger number of input images, $m>5$, and we then need choose the size of the intensity subset. Although a large size implies higher tolerance against noise as long as specular reflection appears just in one frame as assumed above, a crude observation tells that it rather is optimal to keep the size minimum, i.e. four elements, to allow a higher probability of including no specularities. However, the trade-off analysis is also motion dependent and it is beyond the scope of this paper.

\section{Experiments}

We illustrate the performance of the proposed scheme using an object whose surface is moderately glossy (see Figure 2). The input images were taken while the object pose varies under a point light source. As can be observed, specularities appear on different part of the surface according to the object pose relative to the light source. Figure 3 shows the resulting depth map computed for the midmost input image in Figure 2, For comparison, we carried out the depth search 
by three different algorithms within the common framework, i.e., differencing, the geotensity constraint, and the proposed - the geotensity constraint using the intensity subset. In each case we used a $15 \times 15$ template for the search to suppress the error arising from image noise. It turned out that the proposed method allows an improved estimation whereas the other two tend to fail more severely in the region where the surface is either with little texture, or with specularity such as on the top of the belly, although the erroneous estimates in the bottom (conceivably due to complicated inter-reflection) are of an issue for all of them.

\section{Conclusion}

For the problem of 3D object surface reconstruction by a single static camera, we have proposed to relax the condition for geotensity constraint to be applicable to the case that specular reflection is added to the object surface. The key idea is to utilise such a certain subset of intensities that is the least influenced by specular reflection. To our knowledge it is one of the few trials of developing correspondence scheme in the presence of specularity as well as under varying image intensity due to object motion. Although we have illustrated our scheme only for the case of using five input images, the principle also applies to the case that a larger number of images are available. However, we further need some investigations as to how the size of the intensity subset in such cases can be optimally determined, which is one of the issues for future work. Another interesting application will be to generate illumination image basis $[\underline{8}$ in the presence of specular reflection on the basis of the proposed scheme for correspondence.

Acknowledgments. This work is supported in part by MEXT, Japan, under a Grant-in-Aid for Scientific Research (No.16680010) and the research project of MEXT, headed by Professor T. Matsuyama, "Developement of high fidelity digitization software for large-scale and intangible cultural assets".

\section{References}

1. P.A. Beardsley, P. Torr, and A. Zisserman. 3D model acquisition from extended image sequences. In 4rd ECCV, pages 683-695, Cambridge, UK, 1996.

2. P.N. Belhumeur and D.J. Kriegman. What is the set of images of an object under all possible illumination conditions? IJCV, 28:3:245-260, 1998.

3. D. N. Bhat and S. Nayar. Stereo in the presence of specular reflection. In 5 th ICCV, pages 1086-1092, 1995.

4. F. Devernay and O. Faugeras. Computing dierential properties of 3D shapes from stereoscopic images without 3d models. In CVPR, pages 208-213, 1994.

5. P. Fua. Object-centered surface reconstruction: combining multi-image stereo and shading. IJCV, 16:35-56, 1995.

6. B.K.P. Horn. Robot Vision. The MIT Press, 1992.

7. A. Maki, M. Watanabe, and C.S. Wiles. Geotensity: Combining motion and lighting for 3d surface reconstruction. IJCV, 48:2:75-90, 2002.

8. A. Nakashima, A. Maki, and K. Fukui. Constructing illumination image basis from object motion. In 7th ECCV, pages III:195-209, 2002. 
9. A. Pentland. Photometric motion. IEEE-PAMI, 13:9:879-890, 1991.

10. A. Shashua. Geometry and photometry in $3 D$ visual recognition. PhD thesis, Dept. Brain and Cognitive Science, MIT, 1992.

11. D. Simakov, D. Srolova, and R. Basri. Dense shape reconstruction of a moving object under arbitrary, unknown lighting. In 9th ICCV, pages 1202-1209, 2003.

12. C. Tomasi and T. Kanade. Shape and motion from image streams under orthography: a factorization method. IJCV, 9:2:137-154, 1992.

13. M. Weber, A. Blake, and R. Cipolla. Towards a complete dense geometric and photometric reconstruction under varying pose and illumination. In $B M V C$, pages 83-92, 2002.

14. C.S. Wiles, A. Maki, and N. Matsuda. Hyper-patches for $3 \mathrm{~d}$ model acquisition and tracking. IEEE-PAMI, 23:12:1391-1403, 2001. 